\title{
Modeling of Particle Behavior in a Wurster Fluidized Bed: Coupling CFD-DEM with Monte Carlo
}

\author{
Jiang, Z. ${ }^{\text {a,b* }}$; Rieck, C. ${ }^{\text {a }}$; Bück, A. ${ }^{\text {abb }}$ Tsotsas, E. ${ }^{\text {a }}$ \\ a Thermal Process Engineering. Otto von Guericke University Magdeburg, Magdeburg, Germany \\ ${ }^{\mathrm{b}}$ Institute of Particle Technology. FAU Erlangen-Nuremberg, Erlangen, Germany. \\ *E-mail of the corresponding author: zhaochen.jiang@fau.de
}

\begin{abstract}
CFD-DEM approach is applied to investigate circulation motion of particles in a mono-disperse system under both dry and wetting conditions. Good agreement between simulation results and measurement data is observed, in terms of cycle time and residence time in dry condition. The deposition of droplets on the particle surface is modeled by a Monte Carlo approach. The influence of cohesion forces on the macroscopic particle circulation is discussed. In addition, information about coating coverage, the layer thickness and particle size distribution can be predicted by this integrating approach.
\end{abstract}

Keywords: CFD-DEM; Wurster coater; Monte Carlo; cohesion force; residence time. 


\section{Introduction}

Particle coating is widely applied in pharmaceutical, food and fertilizer industry. The Wurster coater can be used as a batch or a continuous fluidized bed to precisely control the quality of the coated product [1]. The entire coating process is considerably complex, caused by a large number of sub-processes, including wetting, drying and film formation; and by the presence of different zones with different controlling parameters (such as gas velocity, gas temperature, and spray rate), as shown in Fig. 1. The enhanced understanding of particle dynamics in different zones is significant to optimize drying kinetics that governs particle formation in coating.

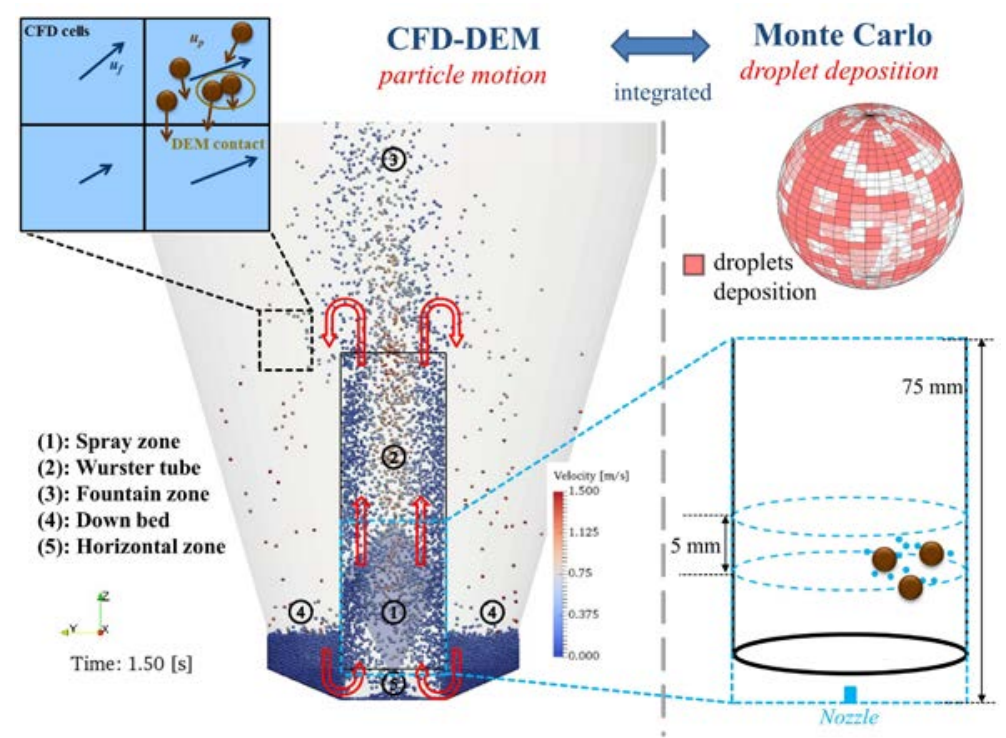

Fig. 1 Modeling of particle behavior by CFD-DEM for particle motion integrating Monte Carlo for droplet deposition (The Wurster coater is divided into 5 process zones; and the droplet deposition takes place in the region marked by the light blue rectangle).

The circulation motion of particles under dry conditions has been investigated in experiments and simulations [2, 3]. However, published studies of the influence of cohesion forces existing in spray zone on the circulation motion are very limited. In this study, computational fluid dynamics-discrete element method (CFD-DEM) was used to investigate the particle motion in the Wurster coater, under both dry and wetting conditions. The influence of cohesion forces, relating to wetting properties and process parameters, on the residence time and the cycle time are discussed based on the analysis of all individual particle trajectories. For the prediction of particle size, a two-zone population balance modeling is usually used to predict the growth of particles during the coating process. In this work, the event of droplet deposition modeled by a Monte Carlo approach [4] was integrated with the particle motion predicted by the CFD-DEM approach, which creates a relatively cost-effective multiscale 
numerical method to predict particle size distribution and coating coverage during the coating process. The effect of cohesion forces in the Wurster tube on particle size distributions for different process times is discussed.

\section{Methodology}

\subsection{CFD-DEM approach}

The CFD-DEM approach has been widely applied to investigate the complex granular flow in chemical applications. The CFD-DEM approach can capture the macroscopic particle dynamics in the multiphase flow, simultaneously providing an insight into behavior of individual particle scale including particle-particle interactions, as shown in Fig. 1. The open source code OpenFoam+LiGGGHTS (CFDEM®project) was used for this study. The detailed governing equations of solid and gas phase, Gidaspow drag model, Hertz soft sphere contact model and rolling model can be found in our previous works [3, 5].

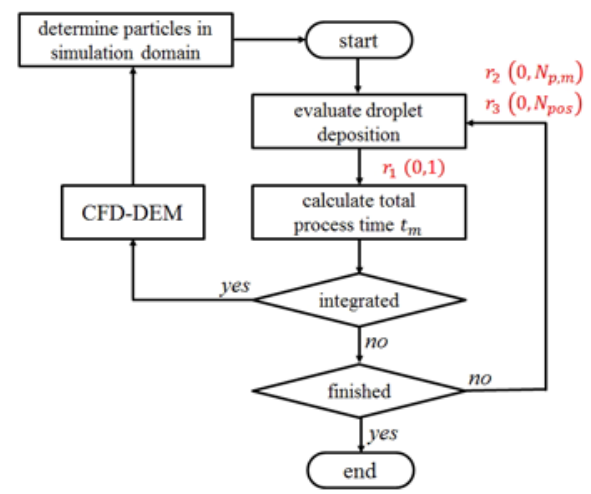

Fig. 2 Flow chart of the Monte Carlo integrated with CFD-DEM including required random numbers ( $N_{p, m}$ is the number of particles in the Monte Carlo domain).

\subsection{Cohesion model}

The capillary force and viscosity force have been implemented into the DEM code [6]. The equation for calculating the capillary force $F_{c}(\mathrm{~N})$ of a specific particle-pair geometry was obtained by fitting the set of discrete solutions of the Laplace equation, expressed as:

$$
F_{c}=\pi \sigma \sqrt{R_{i} R_{j}}\left[C+\exp \left(A \frac{d_{\text {inter }}}{\max \left(R_{i}, R_{j}\right)}+B\right)\right],
$$

where $R_{i}$ and $R_{j}$ are radii of two particles (m), $d_{\text {inter }}$ is the inter-particle distance (m), and $\sigma$ is the surface tension of lqiud $(\mathrm{N} / \mathrm{m})$. The coefficients $A, B$ and $C$ are functions of liquid volume $V_{l}\left(\mathrm{~m}^{3}\right)$, contact angle $\theta$ (radians) and larger particle radius $R_{\max }=\max \left(R_{i}, R_{j}\right)[6]$. The liquid volume between two particles is assumed to be evenly distributed when the interparticle distance is larger than the rupture distance $D_{r}=(1+0.5 \theta) \cdot V_{l}^{1 / 3}(\mathrm{~m})$. 
The viscosity force $F_{v}(\mathrm{~N})$ can be calculated as:

$$
F_{v, n}=6 \pi \mu R^{*} v_{n} \frac{R^{*}}{d_{\text {inter }}}, \quad F_{v, t}=6 \pi \mu R^{*} v_{t}\left[\frac{8}{15} \ln \frac{R^{*}}{d_{\text {inter }}}+0.9588\right],
$$

where $\mu$ is the fluid dynamic viscosity (Pa.s), $R^{*}=R_{i} R_{j} /\left(R_{i}+R_{j}\right)$ is the equivalent radius; and $v_{n}$ and $v_{t}$ are relative velocity of two particles in normal and tangential directions $(\mathrm{m} / \mathrm{s})$, respectively. The capillary force and viscosity force are included into Newton's law of motion for individual particle. Note that these two forces only exist in the Wurster tube and wall boundaries are assumed in dry condition.

\subsection{Monte Carlo approach}

According to the geometry of spray zone, the simulation domain for Monte Carlo is the cylinder with the height of $75 \mathrm{~mm}$ and the radius of the Wurster tube, and the bottom of the domain is aligned with the tip of the spray nozzle, as shown in Fig. 1. The particles in the Monte Carlo domain were determined by the CFD-DEM data. The overview of the Monte Carlo integrated with CFD-DEM is given in Fig. 2. In each Monte Carlo time step $\Delta t_{m}$, one droplet deposition event is guaranteed to happen in the Monte Carlo domain. The time step can be calculated from the number flow rate of droplets injecting into the system, expressed as:

$$
\Delta t_{m}=-\left(\frac{6 \dot{M}}{\pi \rho_{d} d_{d}^{3}}\right)^{-1} \ln r_{1},
$$

where $\dot{M}$ is the mass flow rate of solution and $r_{1}$ is a uniformly distributed random number for the interval $(0,1)$. The droplet diameter is constant. Once the total Monte Carlo process time $t_{m}$ exceeds $0.01 \mathrm{~s}$, CFD-DEM simulation was advanced for $0.01 \mathrm{~s}$ and number of particles in the Monte Carlo domain $N_{p, m}$ was updated based on new CFD-DEM data.

To evaluate the individual droplet deposition, two more random numbers are required: $r_{2}$ to pick up the particle from the domain and $r_{3}$ to choose deposition position on the single particle surface. In current work, each particle in the domain has the same possibility to receive the droplets. Based on the work of Rieck et al. [4], the number of positions (with same size) per particle $N_{\text {pos }}$ is calculated by $N_{\text {pos }}=d_{p}^{2} / d_{\text {contact }}^{2}$. The diameter $d_{\text {contat }}$ is the diameter of contact area, which depends on the contact angle and droplet volume. $N_{\text {pos }}$ was rounded to an integer value in the code. Each position can have four statuses labeled by four numbers in the model: 1) no droplet (initial), 2) with wetting droplet, 3) with dry droplet and 4) no droplet (new). In cases of label 1, 3 and 4, the droplet deposition event can occur. If a wetted position (2) is selected, a new random number $r_{3}$ is generated until the requirement of deposition is satisfied.

The criterion for determining dry or wet position is related to the drying process of the deposited droplet, expressed as: 
Table 1. Summary of the setup for CFD-DEM integrating with Monte Carlo

\begin{tabular}{|c|c|c|}
\hline Parameters & Value & Unit \\
\hline \multicolumn{3}{|c|}{ Particle phase (DEM) } \\
\hline Particle diameter $d_{p, 0}$ & 1.75 & $\mathrm{~mm}$ \\
\hline Particle density $\rho_{d}$ & 1420 & $\mathrm{~kg} / \mathrm{m}^{3}$ \\
\hline Particle number & 50000 & - \\
\hline \multicolumn{3}{|c|}{ Gas phase (CFD) } \\
\hline Gas density $\rho_{g}$ & 1.2 & $\mathrm{~kg} / \mathrm{m}^{3}$ \\
\hline Dynamic viscosity & $1.84 \times 10^{-5}$ & $\mathrm{~Pa} \cdot \mathrm{s}$ \\
\hline Gas flow rate (fluidization $\dot{V}_{g}$ /atomization) & $80.3 / 3.5$ & $\mathrm{~m}^{3} / \mathrm{h}$ \\
\hline Gas temperature & 50 & ${ }^{\circ} \mathrm{C}$ \\
\hline Moisture content of fluidization gas $Y_{\text {inlet }}$ & 1 & $\mathrm{~g} / \mathrm{kg}$ \\
\hline \multicolumn{3}{|c|}{ Liquid phase (Monte Carlo) } \\
\hline Droplet diameter $d_{d}$ & 50 & $\mu \mathrm{m}$ \\
\hline Droplet density $\rho_{d}$ & 1000 & $\mathrm{~kg} / \mathrm{m}^{3}$ \\
\hline Solid density of coating solution $\rho_{s}$ & 1000 & $\mathrm{~kg} / \mathrm{m}^{3}$ \\
\hline Mass flow rate $\dot{M}$ & 0.25 & $\mathrm{~kg} / \mathrm{h}$ \\
\hline Solid mass fraction $\epsilon_{s}$ & 0.3 & - \\
\hline Porosity of coating layer $\varphi_{s}$ & 0.5 & - \\
\hline Liquid content $\alpha_{l}$ & 0.001 & - \\
\hline Surface tension $\sigma$ & 0.072 & $\mathrm{~N} / \mathrm{m}$ \\
\hline Contact angle $\theta$ & 30 & $\circ$ \\
\hline Liquid viscosity $\mu$ & $10^{-4}$ & $\mathrm{~Pa} \cdot \mathrm{s}$ \\
\hline \multicolumn{3}{|c|}{ CFD-DEM simulation parameters } \\
\hline CFD time step & $5 \times 10^{-5}$ & $\mathrm{~s}$ \\
\hline CFD cell number (structured hexahedral) & 81600 & - \\
\hline DEM time step & $10^{-5}$ & $\mathrm{~s}$ \\
\hline Integrate time Monte Carlo and CFD-DEM & 0.01 & s \\
\hline Simulation time & 20 & $\min$ \\
\hline
\end{tabular}

$$
t_{m} \geq t_{\text {deposition }}+\Delta t_{\text {drying }},
$$

where $t_{\text {deposition }}$ is the moment the droplet deposition happens, and $\Delta t_{\text {drying }}$ is the drying time of the deposited droplet. Considering the first drying period only, the $\Delta t_{\text {drying }}$ can be calculated by [4]:

$$
\Delta t_{\text {drying }}=\frac{\left(\frac{1}{6} \pi d_{d}^{3}\right) \cdot \rho_{d} \cdot\left(1-\epsilon_{s}\right)}{\beta_{m} A_{\text {dep }} \rho_{g}\left(Y_{\text {sat }}-Y\right)},
$$

where $\beta$ is the mass transfer coefficient ( $\mathrm{m} / \mathrm{s}), Y_{\text {sat }}$ is the adiabatic saturation moisture content of fluidization gas (g liquid/kg dry gas), and $Y=Y_{\text {inlet }}+\dot{M} \cdot\left(1-\epsilon_{s}\right) /\left(\dot{V}_{g} \cdot \rho_{g}\right)$ is the moisture content of bulk gas. $A_{\text {dep }}$ is the curved area of deposit droplet in contact with the gas $\left(\mathrm{m}^{2}\right)$, which can be calculated by: 


$$
A_{\text {dep }}=\frac{1}{2} \frac{\pi \cdot d_{\text {contact }}^{2}}{1+\cos \theta} .
$$

The thickness of solid layer in single position on particle surface $h_{i}$ can be calculated as:

$$
h_{i}=\left(\frac{d_{\text {core }}^{3}}{8}+\frac{3}{4} \frac{N_{\text {pos }} \cdot\left(\frac{1}{6} \pi d_{d}^{3}\right) \cdot \frac{\rho_{d} \cdot \epsilon_{s}}{\rho_{s} \cdot \varphi_{s}}}{\pi}\right)^{1 / 3}-d_{\text {core }}
$$

where $d_{\text {core }}$ is the diameter of core particle. The coating coverage $\Psi$ can be evaluated by:

$$
\Psi=\frac{N_{\text {pos }, \text { tot }}-N_{\text {pos, free }}}{N_{\text {pos }, \text { tot }}},
$$

where $N_{\text {pos.tot }}$ and $N_{\text {pos.free }}$ are total number of positions and number of positions without droplet, respectively. With the average coating thickness $h_{m}$, the particle diameter can be expressed as: $d_{p}=d_{\text {core }}+2 h_{m}$.

\subsection{Simulation setups}

The mesh of Wurster coater was built by O-grid method [3], according to the configuration used in PEPT experiments [2]. The initial particle diameter $d_{p, 0}$ is $1.75 \mathrm{~mm}$. All important simulation parameters in the sub-models of CFD-DEM integrating with Monte Carlo are summarized in Table 1. The coupling interval between DEM and CFD is 100 time steps of DEM; and the integrating interval with Monte Carlo is $0.01 \mathrm{~s}$, which is approximately $2 d_{p, 0} / v_{m}$ (mean particle velocity in the Wurster tube).

\section{Results and discussion}

\subsection{Particle circulation motion}

As shown in Fig. 3, the global circulation of particle from simulation with cohesion forces $(0.1 \%)$ is very similar to that in dry condition; however, the particles tend to be clustered in the Wurster tube. The detailed value of mean cycle time and mean residence time are listed in Table 2. The ideal cycle time and residence time in the Wurster tube were found to be in good agreement with PEPT measurement data in dry condition. However, the non-ideal cycle was underestimated in the simulation. With the effect of cohesion forces, the ideal cycle time and the fractions of ideal cycle are decreased, resulting in the increase of total cycle time. The decrease of the fractions of ideal cycle may cause by upwards and downwards motion of particle clustering in the Wurster zone.

\subsection{Particle coating}

Figure 4 left) shows the coating coverage of a sample particle after $70 \mathrm{~s}$ and the spherical particle was mapped into 2D space based on number of deposition positions. The gray level 

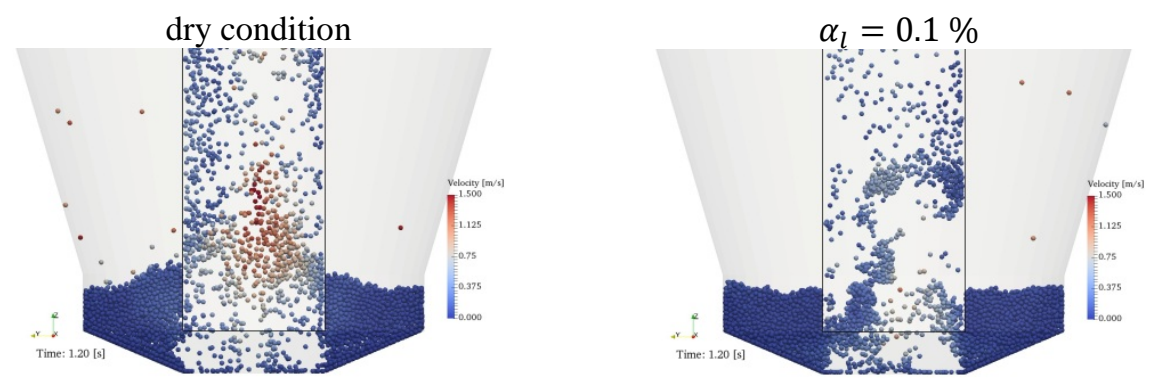

Fig. 3 Influence of cohesion forces on particle motion in the Wurster coater.

Table 2. Comparison of ideal cycle time, overall cycle time, residence time

\begin{tabular}{lccc}
\hline \multirow{2}{*}{ Variable } & \multicolumn{2}{c}{ CFD-DEM simulation } & Measurement [2] \\
\cline { 2 - 4 } & \multicolumn{1}{c}{$d r y$} & $\alpha_{l}=0.1 \%$ & $d r y$ \\
\hline $\bar{t}_{i c}[\mathrm{~s}]$ & $4.98(52.8)$ & $4.25(33.5)$ & $4.84(99.0)$ \\
$\bar{t}_{c}[\mathrm{~s}]$ & $5.82(61.5)$ & $6.42(73.3)$ & $6.14(89.8)$ \\
$r_{n}[\%]$ & 78.3 & 60.2 & 55.3 \\
$\bar{t}_{r, t}[\mathrm{~s}]$ & $0.96(33.7)$ & $0.90(44.6)$ & $1.00(-)$ \\
$\bar{t}_{r, s}[\mathrm{~s}]$ & $0.15(22.1)$ & $0.14(28.3)$ & - \\
\hline
\end{tabular}

$* \bar{t}_{i c}$ is mean ideal cycle time, $\bar{t}_{i c}$ is mean total cycle time, $r_{n}$ is number ratio of ideal cycle, $\bar{t}_{r, t}$ and $\bar{t}_{r, t}$ are mean residence times in Wurster tube and spray zone; the coefficient of variation $(\mathrm{CV})$ is in the brackets.
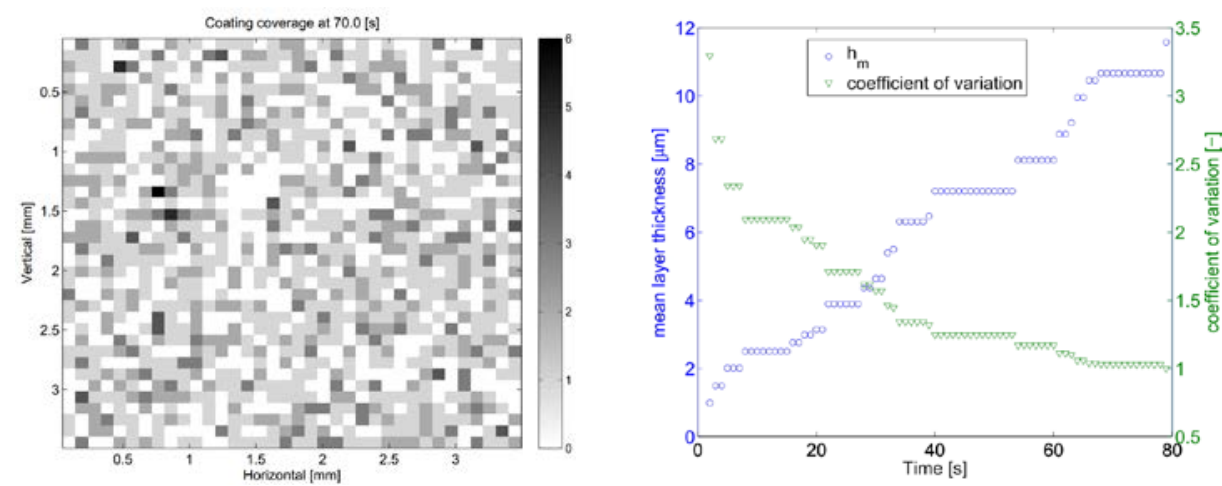

Fig. 4 Coating coverage and layer thickness of sample particle from wetting simulation: left) droplet deposition on single particle, right) layer thickness and $C V$ with respect to time.

represents the number of deposition droplets in each pixel. According to Eq. (8), the coating coverage at this moment is $67 \%$. However, the coating coverage cannot roundly measure the uniformity of coating layer. The coefficient of variation (the ratio of standard deviation to mean) of the layer thickness is 1.05 at this moment. Figure 4 right) shows that the mean layer thickness increases and the coefficient of variation decreases. The variation only happens when the particle passes through the Wurster tube. Figure 5 show the size distributions under dry and wetting conditions. There are wider distributions of particle diameter $d_{p}$ for simulations with cohesion forces for different process times. 


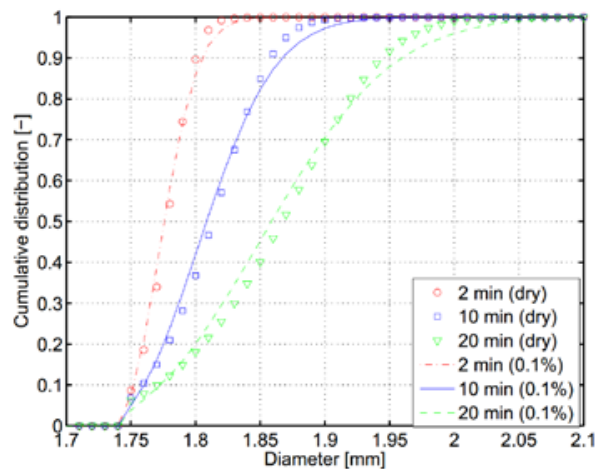

Fig. 5 Particle size distributions of 50000 particles in different process time (both dry and wetting).

\section{Conclusion}

Cohesion forces scatter distributions of cycle time, residence time and particle size. It is important to provide enough drying capacity in fluidization gas to prevent the appearance of particle agglomeration and achieve high product quality in the Wurster coating process.

\section{Acknowledgements}

The authors gratefully acknowledge the funding of this work by the German Federal Ministry of Science and Education (BMBF) as a part of the InnoProfileTransfer project NaWiTec (03IPT701X).

\section{References}

[1] Tsotsas, E. Multiscale approaches to processes that combine drying with particle formation. Drying Technology 2015, 33, 1859-1871.

[2] Li, L.; Rasmuson, A.; Ingram, A.; Johansson, M.; Remmelgas, J.; von Corswant, C.; Folestad, S. PEPT study of particle cycle and residence time distributions in a Wurster fluid bed. AIChE 2015, 61, 756-768.

[3] Jiang, Z.; Bueck, A.; Tsotsas, E. CFD-DEM study of residence time, droplet deposition, and collision velocity for a binary particle mixture in a Wurster fluidized bed coater. Drying Technology 2018, 36(6), 638-650.

[4] Rieck, C.; Bück, A.; Tsotsas, E. Monte Carlo modeling of fluidized bed coating and layering processes. AIChE J 2016, 62, 2670-2680.

[5] Jiang, Z.; Hagemeier, T.; Bueck, A.; Tsotsas, E. Color-PTV measurement and CFDDEM simulation of the dynamics of poly-disperse particle systems in a pseudo-2D fluidized bed. Chemical Engineering Science 2018, 179, 115-132.

[6] Soulié, F.; Cherblanc, F.; El Youssoufi, M.; Saix, C. Influence of liquid bridges on the mechanical behaviour of polydisperse granular materials. International Journal for Numerical and Analytical Methods in Geomechanics. 2006, 30, 213-228. 